\title{
Development of Rapid Diagnostic Technology for Pig Disease (2) - Rapid detection of PPE in the pig feces -
}

\author{
Hyuck-Joo Kim ${ }^{1}$, Jong-Tae Hong ${ }^{1}$, Byeong-Kee ${ }^{1}{ }^{1}$, Giyoung Kim ${ }^{1}$, Suk Kim ${ }^{2}$ \\ ${ }^{1}$ National Academy of Agricultural Science, RDA., Suwon, Korea \\ ${ }^{2}$ College of Veterinary medicine, Gyeongsang National University, Jinju, Gyeongnam, Korea
}

Received: May $1^{\text {st }}, 2013$; Revised: May 23 ${ }^{\text {th }}, 2013$; Accepted: May 29 2013

\section{Abstract}

Purpose: Porcine proliferative enteropathy (PPE), caused by the obligate intracellular bacterium Lawsonia intracellularis, is a widely distributed disease throughout the world causing substantial economic loss. In order to diagnose PPE rapidly, the rapid kit was developed and tested. Methods: In this study, a rapid kit was developed to screen the PPE rapidly at the pig farm. Also, occult blood test with fecal occult blood (FOB) kit was done for detecting the blood in pig feces which might be the evident of hemorrhagic PPE. For developing the kit, we tested fecal samples of PPE infected pigs diagnosed by polymerase chain reaction (PCR) method. Results: With the developed rapid kit, Lawsonia intracellularis was detected in high density emulsion of ileum. On the other hand, the test result of detecting Lawsonia in feces showed too high non-specific response. In addition, nevertheless the FOB test result showed that blood evident could be founded in pig feces, the diagnosing result was not fit to PCR test result, which shows blood in pig feces could be from not only hemorrhagic PPE but also many reasons. Conclusions: To deal with the PPE effectively, it will be better for farmers to screen the PPE in earlier stage with easy and rapid diagnosing tool on farm. This study found out that the rapid kit could detect the Lawsonia intracellularis and hemoglobin in pig feces. However, the non-specific response to negative samples of PPE was too high to use at a pig farm. Further research is needed for lowering the non-specific response with the rapid kit.

Keywords: FOB, Lawsonia, Pig feces, Porcine proliferative enteropathy (PPE), Rapid kit

\section{Introduction}

The previous study (Kim et al., 2012) explored the combination of antibodies and the diagnosis of the porcine proliferative enteropathy (PPE) using the polymerase chain reaction (PCR) test for developing lateral flow immunoassay.

The PPE infection was serious in that $25 \%$ of the samples used in the previous study were infected. According to Chung (2008), 35.2\% of the 155 samples were infected, and $53.2 \%$ of pig farms in Gyeong-nam province, Korea were infected. Monitoring possible infectious

\footnotetext{
*Comesponding author: Hyuck Joo Kim

Tel: +82-31-290-1867; Fax: +82-31-290-1860

E-mail: agrihj@rda.go.kr
}

diseases and preventing the spread of the infections at pig farms are important to reduce the risk of developing diseases. However, conventional lab tests such as PCR test, fluorescence antibody test, and immunohistochemistry test take several days for the results. For this reason, those tests fail to response effectively and farmers treat the diseases relying on the visual clues. Therefore, development of rapid kit, lateral flow immunoassay, is needed to diagnose the diseases and to prevent the spread of the infection.

Impedance biosensor, enzyme-linked immunosorbent assay (ELISA) test kit, and rapid lateral flow immunoassay (rapid kit) were used to detect the infectious bacterials. For detecting the infectious salmonella, a rapid kit was developed (Kim and Lee, 2011). Impedance biosensor 
and ELISA test kit need the lab work, so famers cannot use them instantly. Therefore, this study will use the rapid kit to diagnose the PPE and present the results of the experiments.

The objective of this study was to develop or test lateral flow rapid kits for detecting the PPE. The study was consisted of 1) manufacturing and testing the rapid kit for detecting Lawsonia intracellularis 2) detecting blood in the feces which indicates acute hemorrhagic PPE using FOB test kit.

\section{Materials and Methods}

PPE infected pigs have two kind of evident in the feces, which are bacterials of Lawsonia intracellularis and hemoglobin of blood from the ileum. Accordingly, in this study, detection test for Lawsonia intracellularis was done with the manufactured rapid kit, and detecting blood in the feces which indicates acute hemorrhagic PPE was done with the FOB test kit.

\section{Preparation of the antibodies and samples}

Antibody is an important component for detecting target antigen, so it should be supplied at affordable prices. No antibody for the rapid kit has been developed yet; therefore, self-made serums to detect Lawsonia intracellularis or commercially available antibodies for immunohistochemistry test are the only option.

In 2010 2012, the antibodies to detect the target bacterial of Lawsonia intracellularis was manufactured with the cooperation of College of Veterinary Medicine, Gyeongsang National University as the previous study (Kim et al., 2012). Table 1 showed the concentration of the each antibody used in this study.

Experiment was conducted to detect Lawsonia intracellularis which causes PPE. Liquid emulsion of ileum and pretreated feces containing Lawsonia intracellularis from the PPE infected pigs, produced by College of Veterinary Medicine, Gyeongsang National University, were used as the antigen. The lab of College of Veterinary Medicine produced the liquid emulsion of ileum three times. While the concentration of Lawsonia intracellularis differs as the state of the liquid emulsion of ileum at the time of manufacture, so the concentration of Lawsonia intracellularis in the liquid emulsion of ileum was assumed as $10^{4} \sim 10^{6} \mathrm{cfu} / \mathrm{ml}$.

As referred in the previous study (Kim et al., 2012), 200 samples were collected from a pig farm in Gyeong-nam province, Korea, and tested by the PCR method. The results of $1^{\text {st }}$ experiment showed $25 \%$ infection rate.

The samples, 200 samples were collected from pig barns and slaughterhouses in Gyeongnam province, Korea, used in $2^{\text {nd }}$ experiment, showed $14 \%$ infection rate because 23 fecal samples out of 200 were positive. Then, 10 fecal samples ( 4 positive, 6 negative) from three pig farms in Gyeongnam province, Korea,each were prepared for $3^{\text {rd }}$ experiment (Kim et al., 2012).

\section{Manufacturing the rapid kit for detecting Lawsonia intracellularis}

The rapid kit of lateral flow immunoassay had four porous membranes or pads as in Figure 1. Sample pad, conjugation, nitrocellulose membrane, and absorbent pad were on the strip, and buffer reagents were pretreated on the sample pad. A conjugate (a complex of antibody-gold particles) was applied on the conjugate pad to identify the target with color change.

The gold particles and antibodies were conjugated following instructions of the manufacturer's protocol. After the $\mathrm{pH}$ testing, the optimum $\mathrm{pH}$ for conjugating the antibody with gold was selected. Two lines were fixed on

Table 1. The concentration of the polyclonal antibodies

\begin{tabular}{cccccc} 
& & \multicolumn{3}{c}{ Manufactured } & \multicolumn{2}{c}{ Imported from Japan } \\
\cline { 3 - 6 } Antibody & & LsaA-R1 & LsaA-R2 & LsaA-Iw & Bio 323 \\
\cline { 3 - 6 } & & Polyclonal & Polyclonal & Polyclonal & Monoclonal \\
\cline { 3 - 6 } $\begin{array}{c}\text { Concentration } \\
(\mathrm{mg} / \mathrm{ml})\end{array}$ & 1 & 6.50 & 5.82 & 5.82 & - \\
& avg & 6.44 & 5.65 & 5.65 & 0.2 \\
Remarks & & 6.47 & 5.74 & 5.74 & Manufacturer's \\
specifications
\end{tabular}




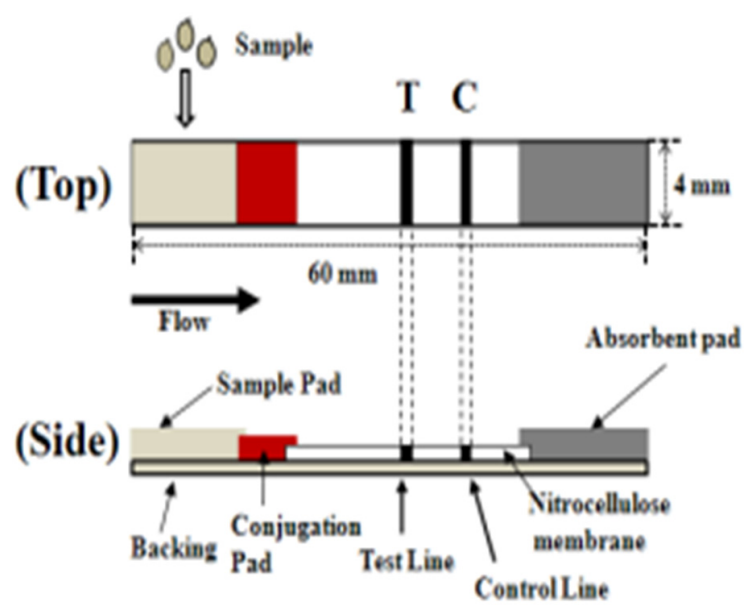

Figure 1. Schematic of rapid kit.

(Top)

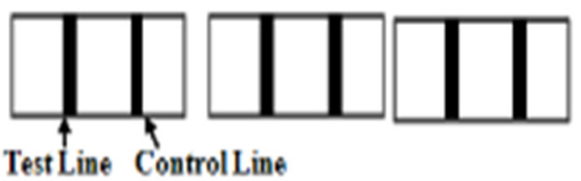

(Side)

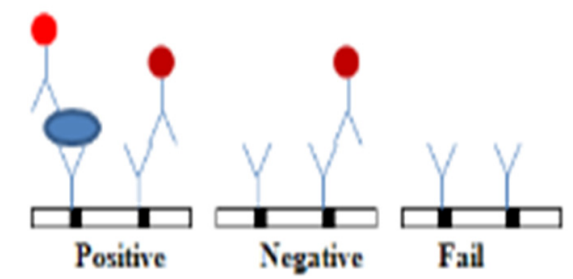

Figure 2. The test result.

the nitrocellulose membrane: one was a test line which contained antibody for capturing the Lawsonia intracellularis and the other was a control line which indicated the success or failure of the experiment. The absorbent pad was used as the reservoir of the wasted samples.

All membranes and pads were from Millipore Corporation (USA). The anti-rabbit IgG was from Pierce Biotechnology INC (USA) and reagents such as phosphate buffer solution (PBS) were from Sigma Corporation (USA). Antibodies on the conjugation pad and test line used Lawsonia intracellularis. Figure 2 showed the test results of the rapid kit of lateral flow immunoassay.

The experiment was conducted to detect Lawsonia intracellularis with liquid emulsion of ileum and pretreated feces using rapid kit. After $1 \mu \mathrm{l}$ of the pretreated feces was dyed with Uranyl Acetate, a complex of antibodygold particles was applied in the feces. Transmission Electron Microscope (LE0912AB, Carl Zeiss, Germany) with $100 \mathrm{kV}$ in Figure 3 was used to check the Lawsonia intracellularis in the feces.

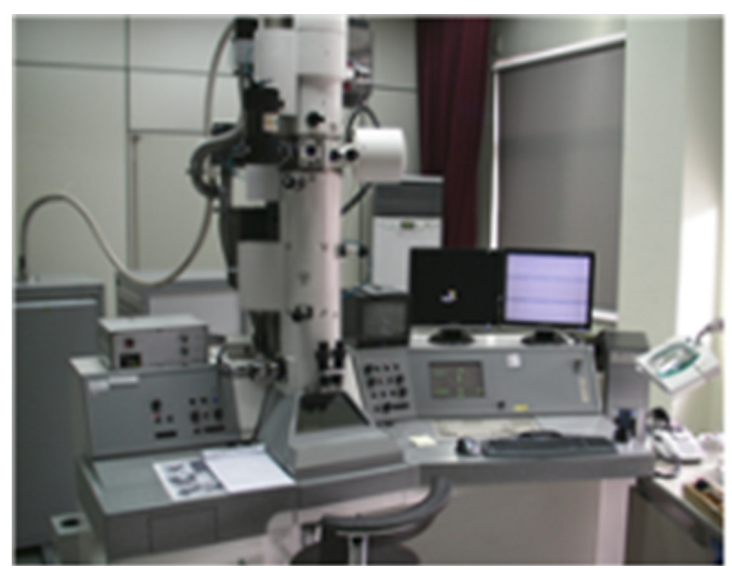

Figure 3. Transmission Electron Microscope.

\section{Detecting PPE using fecal occult blood (FOB) test kit}

The PPE caused by the Lawsonia intracellularis has two major clinical aspects, the acute hemorrhagic form and a chronic form. The acute hemorrhagic form could be diagnosed early if hemoglobin is detected in the feces. Fecal occult blood (FOB) test kit, originally developed for detecting blood from human feces, was used to detect hemoglobin for this study. When the concentration of the hemoglobin is over $0.5 \mathrm{mg} / \mathrm{ml}$, the sandwich pair test detects the hemoglobin. $100 \mu \mathrm{l}$ of the sample was dropped in the kits with pipette, and five minutes later the color changed in the kits. Then, scanning electron microscope was used to identify the red blood cell on the conjugation pad.

\section{Detecting PPE in the pig farm}

The experiments described above were conducted in the lab; however, it was not easy to conduct them in the pig farms due to the pretreatment of the feces. Therefore, the study had experiments with the feces in the pig farm without the pretreatment. A fecal sample was collected with sampling rod and placed in a tube containing surfactants. The mixture in the tube was shaken well, then, it was dropped on the kit. Five minutes later the line was detected.

\section{Results and Discussion}

\section{Test results of rapid kit}

Sandwich combination of Lawsonia intracellularis with 
antibody following ELISA protocol (Young In Frontier co., ltd, 2010) was discussed in the previous study (Kim et al., 2012). It is not easy to make ELISA kit using collected antibodies, but sandwich reaction with the combination of the antibodies can be identified. Therefore, this sandwich reaction was used in developing the rapid kit. Antigenantibody reaction was observed in the experiments for developing the rapid kit as in ELISA test (Kim et al., 2012).

\section{Manufacturing the rapid kit for detecting Lawsonia intracellularis}

\section{Conjugation of antibody-gold particles}

The antigen in the rapid kit was detected by the color change of gold particles in the conjugation which followed instruction of the Bioassay protocol. The conjugation test result revealed that the optimum $\mathrm{pH}$ for conjugation of antibody with gold was 5.4 9.2 as shown in Figure 4, which didn't indicate any color changes and sediments. However, the color of some of the conjugation with Bio

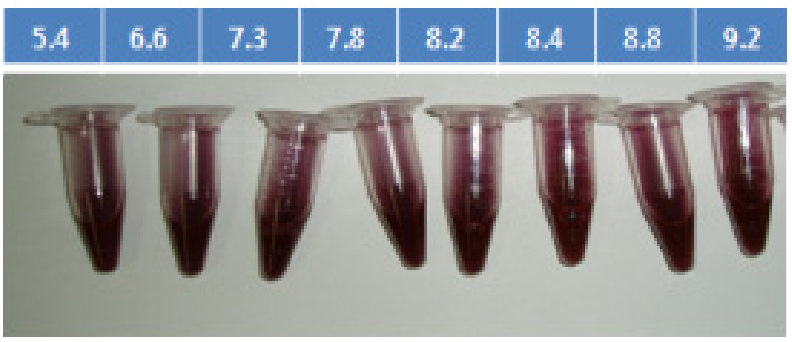

Figure 4. Conjugation of antibody-gold particles.

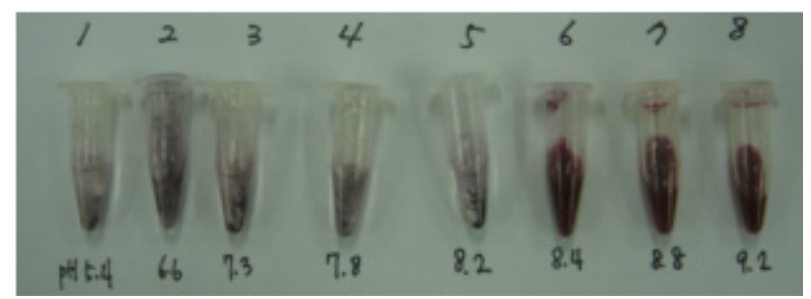

Figure 5. Conjugation of antibody-gold particles with $\mathrm{pH}$ adjusting.
323 antibody turned purple with sediments and the conjugation was not successful. As shown in the Figure 5, only 3 samples of $\mathrm{pH} 8.4,8.8$, and 9.2 showed the proper conjugations.

\section{Test results using polyclonal antibody}

Table 2 showed the test results using polyclonal antibody manufactured for this study. The kit used polyclonal antibody of LsaA-R1 as a detector and LsaA-R2 as a capture. Liquid emulsion of ileum was used as an antigen. No positive sample was shown with the polyclonal antibody, and no sandwich combination was observed.

\section{Test results using polyclonal antibody and monoclonal antibody}

Antibody in the rapid kit is important, and the previous study (Kim et al., 2012) found that polyclonal antibody LsaA-JP as a detector and monoclonal antibody Bio 323 as a capture was the best. The test kit for this study was modified in that the width of the kit was narrowed to 5 $\mathrm{mm}$ to improve the flow of the sample.

The experiment started with $100 \mu$ drop of the sample, liquid emulsion of ileum with concentration of $10^{4} \sim 10^{6}$ $\mathrm{cfu} / \mathrm{ml}$, to the inlet of sample pad, and five minutes later the line was detected as in Figure 6. In the experiment

\section{$\begin{array}{ll}10^{5} \sim 10^{6} \mathrm{cfu} / \mathrm{al} & 10^{4} \sim 10^{5} \mathrm{cfu} / \mathrm{hl}\end{array}$}

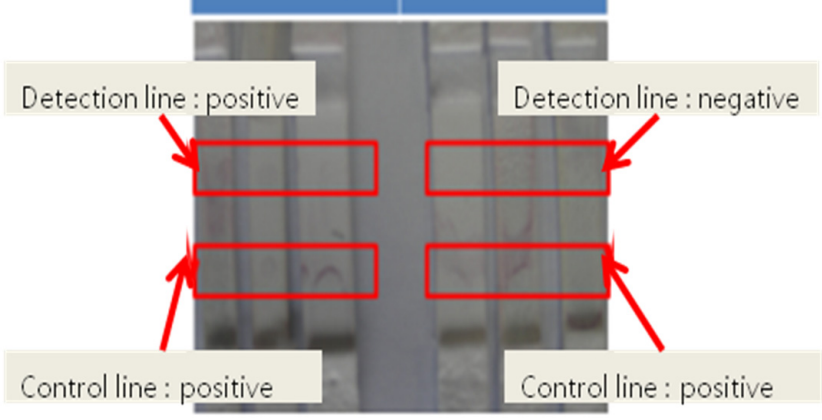

Figure 6. Test result using liquid emulsion of ileum.

Table 2. Test results of combination of LsaA-R1 and LsaA-R2 (+ : positive, - : negative)

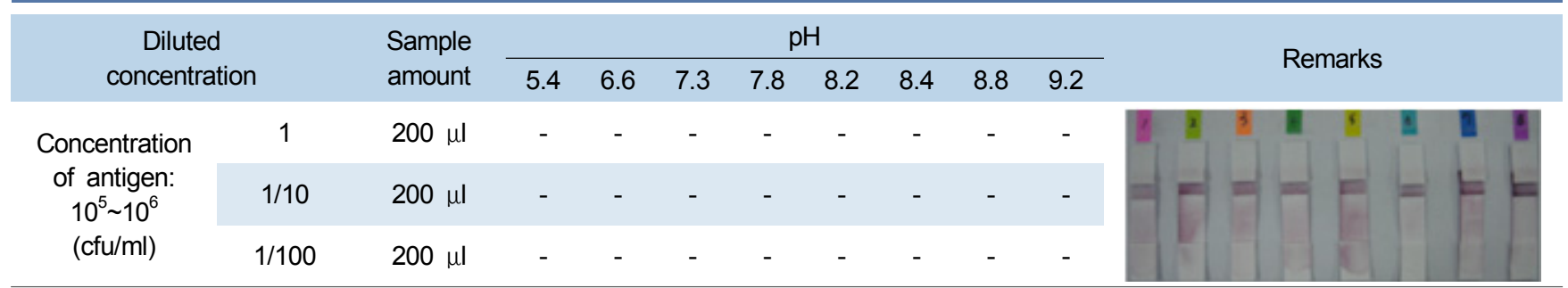


Kim et al. Development of Rapid Diagnostic Technology for Pig Disease (2) - Rapid detection of PPE in the pig feces Journal of Biosystems Engineering • Vol. 38, No. 2, 2013•www.jbeng.org

Table 3. Test result of feces with concentration of antigen of $10^{5} \sim 10^{6} \mathrm{cfu} / \mathrm{ml}$

\begin{tabular}{|c|c|c|c|c|c|c|c|c|c|c|c|c|c|c|c|c|}
\hline & \multicolumn{9}{|c|}{ Test result } & \multirow{2}{*}{\multicolumn{7}{|c|}{ Remarks }} \\
\hline & \#1 & \#2 & \#3 & \#4 & \#5 & \#6 & \#7 & \#8 & \#9 & & & & & & & \\
\hline (100 $\mu \mathrm{l})$ & + & + & - & - & + & + & + & - & - & & 7 & $\frac{79}{4}$ & 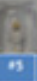 & 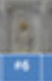 & & $n$ म \\
\hline Response & + & + & + & + & + & + & + & + & - & & & & & & & \\
\hline $\begin{array}{l}\text { Response } \\
\text { time (min) }\end{array}$ & 5 & 5 & 5 & 5 & 5 & 5 & 5 & & 5 & & & & & & & \\
\hline
\end{tabular}

with concentration of antigen of $10^{5} \sim 10^{6} \mathrm{cfu} / \mathrm{ml}$, the test line showed positive to the Lawsonia intracellularis. However, lower concentration of antigen of $10^{4} \sim 10^{5} \mathrm{cfu} / \mathrm{ml}$ showed negative to the Lawsonia intracellularis. From these results, we discovered that the Lawsonia intracellularis may not be detected in the feces with low concentration.

Liquid emulsion of PPE infected ileum was used in detecting the Lawsonia intracellularis with the rapid kit. However, feces sample should be used to detect the Lawsonia intracellularis at a pig farm, so the pretreated feces were used in this experiment. Sample with low concentration of antigen showed no positive reaction, and sample with concentration of antigen of $10^{5} \sim 10^{6}$ $\mathrm{cfu} / \mathrm{ml}$ showed non-specific reactions on the test line as in Table 3.

The experiment had some modification because the color change of the gold particle was not good, and the test line and the control line were not clear for detecting due to the uneven concentration of antigen. Thus, the sample pad and conjugation pad were pretreated with the conditions of $0.1 \mathrm{M}$ Tris, $0.5 \%$ casein, $1.0 \%$ Triton-X $100,0.1 \% \mathrm{NaN}_{3}$, and $\mathrm{pH} 8.5$. In this test, the positive and negative fecal samples, the condensed vaccine (Boehringer Ingelheim Inc.) and control with buffer was used as antigens. Figure 7 shows the test result. The result turned out that the Lawsonia intracellularis was not detected. This experiment found that rapid kit could not detect the Lawsonia intracellularis at the pig farm.

Figure 8 shows the bacterium and gold particles in the feces through the Transmission Electron Microscope, and Figure 9 show the Lawsonia intracellularis with conjugated gold particles (Guedes et al., 2003). The bacterium in Figure 8 was similar with the Lawsonia intracellularis. The size of the gold particle was $40 \mathrm{~nm}$, and it was attached to the bacterium. However, it was hard to name the bacterium in Figure 8 as the Lawsonia intracellularis because the Lawsonia intracellularis has gold particles outside of it as Figure 9.

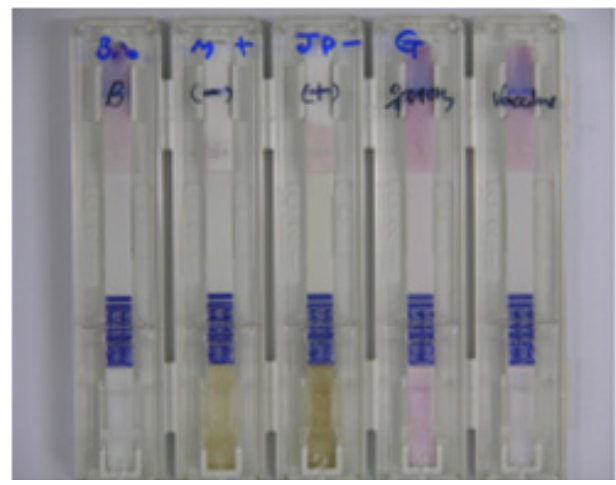

Figure 7. Test result with buffer, negative/positive sample, liquid emulsion of ileum, and Lawsonia vaccine.

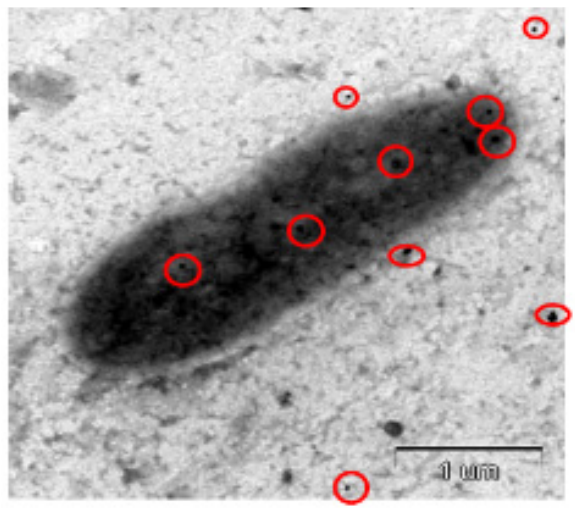

Figure 8. Bacterium and gold particles $(\mathrm{O})$ in the feces.

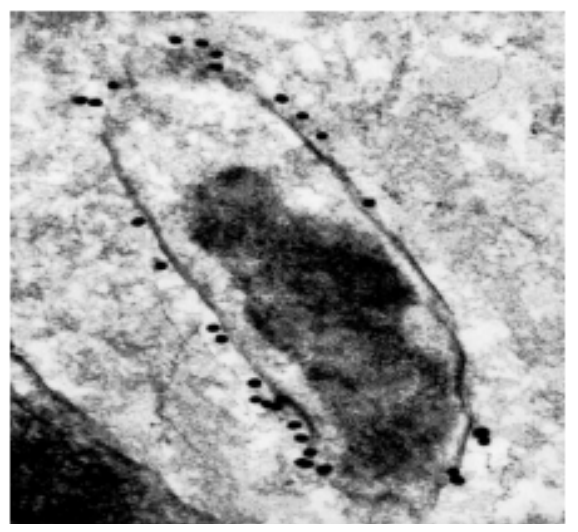

Figure 9. Lawsonia intracellularis with conjugated gold. 


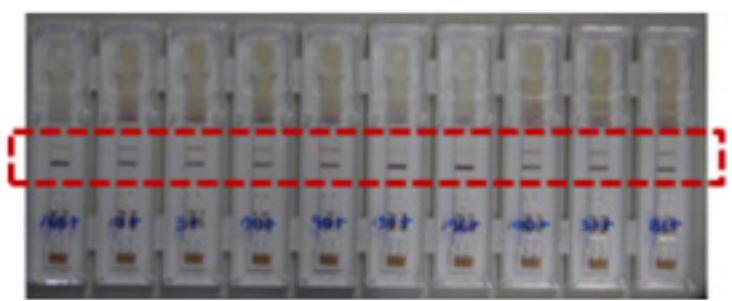

Figure 10. FOB test results.

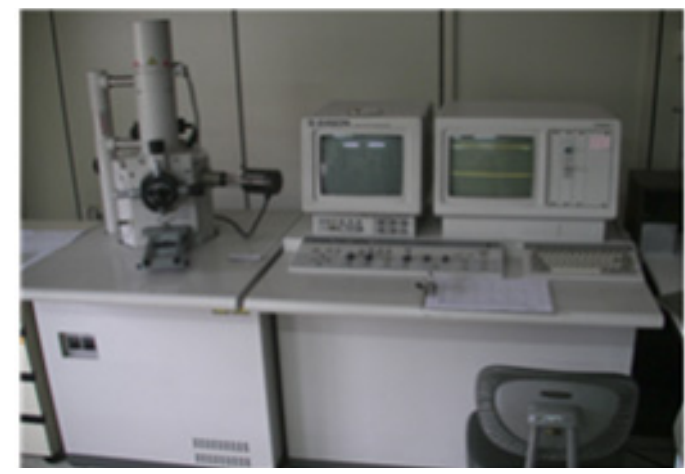

Figure 11. Electron microscope (SEM).

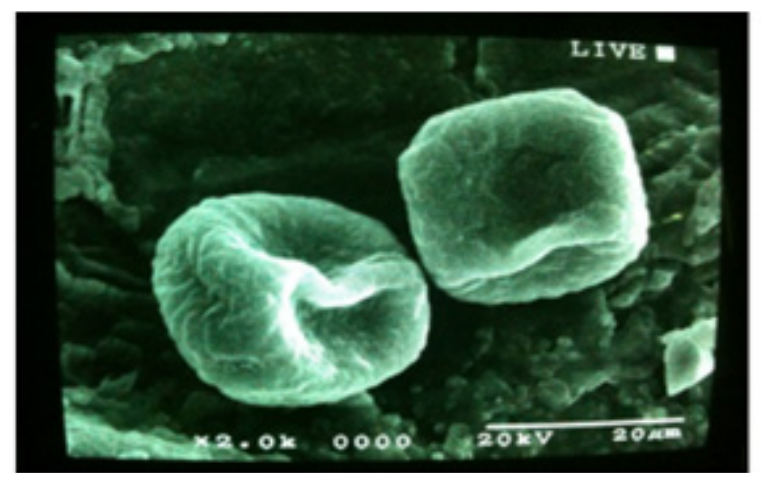

Figure 12. Red blood cells found on the conjugation pad.

\section{Experiment for detecting hemorrhagic PPE using FOB test kit}

First experiment for detecting hemorrhagic PPE using FOB test kit

The results of the first experiment for detecting hemorrhagic PPE using FOB test kit presented in Figure 10. The 200 pretreated fecal samples from the pigs diagnosed as PPE by PCR test at Gyeongsang National University were used as the antigen. The results of the experiment agreed $90 \%$ with the test results from the PCR test. Therefore FOB test assumed to be used to diagnose hemorrhagic PPE.

The red blood cells in the feces used for the experiment

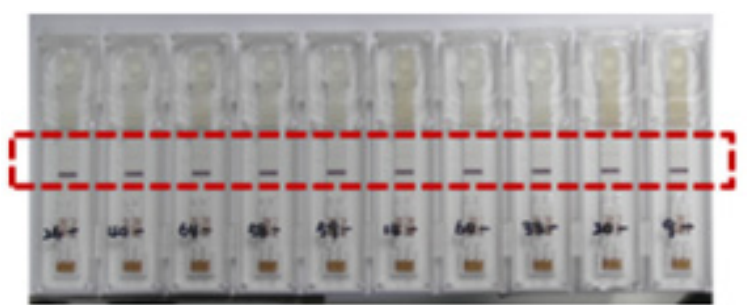

were extracted to double check the results. An scanning electron microscope (S-2460N, Hitachi, Japan) shown in Figure 11 was used, and the red blood cells were found on the conjugation pad as in Figure 12. In addition, field test was conducted to detect hemorrhagic PPE in feces. 20 positive samples and 20 negative samples identified by PCR test showed $90 \%$ concordance rate with the lab test.

\section{Second experiment for detecting hemorrhagic PPE using FOB test kit}

The first experiment used the samples from one pig farm, so various samples were needed to verify the first experiment. The samples for the second experiment were collected from the slaughterhouses in Gyeongnam province with the cooperation of College of Veterinary Medicine, Gyeongsang National University. 86 out of 200 samples diagnosed as PPE by the PCR test were used.

Test results showed 15 out of 18 samples were agreed with the result of PCR test in PCR positive samples, and concordance rate was $83 \%$. 30 out of 68 samples were agreed with the result of PCR test in PCR negative samples, and non-specific response was $56 \%$. The high non-specific response was due to the blood in the feces, because the blood could be caused by other bacteria besides PPE. Accordingly, third experiment with various samples was needed to verify the second experiment.

Third experiment for detecting hemorrhagic PPE using FOB test kit in other three pig farms

The samples for the third experiment were collected from the pigs suspected with PPE at several pig farms in Gyeongnam province.

The feces were PCR tested and used for the third experiment. The results of the experiment shows 5 out of 12 samples were agreed with the result of PCR test in PCR positive samples, and concordance rate was $42 \% .17$ out of 18 samples were agreed with the result of PCR test in PCR negative samples, and non-specific response was 
$6 \%$. The non-specific response in the negative samples was low, but the concordance rate of positive samples was also low. For this reason, it was difficult to derive a clear correlation between the positive or negative to PPE and detection of fecal occult blood.

The results showed high concordance rate and nonspecific response in each pig farm. It was due to the uncertainty of the correlation between the swine diagnosed by PPE and hemorrhage. In addition, hemorrhage could be accompanied by swine dysentery or salmonella infection besides PPE. Therefore, FOB test kit diagnosed the hemorrhagic PPE in a single pig farm. However, samples from various environments such as several pig farms, or slaughterhouses could not be diagnosed PPE by detecting hemoglobin.

\section{Conclusions}

(1) Rapid kit of lateral flow immunoassay with sandwich combination of antibody-antigen was developed to detect Lawsonia intracellularis. The rapid kit had four porous membranes or pads: a sample pad where buffer reagents were pretreated, a conjugation pad where a complex of antibody-gold particle was applied to identify the color change of the target, a nitrocellulose membrane which had a test line and a control line, and an absorbent pad to store the wasted samples.

(2) The different types of antibody were needed depending on the antigen, therefore, self-made polyclonal antibody (LsaA-R1, R2), polyclonal antibody imported from Japan (LsaA-JP), and monoclonal antibody imported from Europe (Bio 323) were used for this study. ELISA test was performed to check the combination precisely, and the pair of Bio 323 monoclonal antibody and LsaA-JP polyclonal antibody showed the sandwich. However, optical density value was high without the antigen; it was not suitable for developing ELISA kit.

(3) Self-made antibody (LsaA-R1, R2) did not react with the liquid emulsion of ileum, and antibody imported from Japan (LsaA-R1, LsaA-JP) reacted slightly with it, which was not enough to detect. In the experiment of antibody imported from Japan and Europe (LsaA-JP, Bio 323), $10^{5} \sim 10^{6} \mathrm{cfu} / \mathrm{ml}$ of antigen concentration was reacted on the test line. Consequently, Lawsonia intracellular can be detected in the combination of polyclonal antibody and monoclonal antibody. However, the low concentration of Lawsonia intracellular is in the feces cannot be detected, because $10^{4} \sim 10^{5} \mathrm{cfu} / \mathrm{ml}$ of antigen concentration did not show the positive reaction. Therefore, we concluded that samples with low concentration of antigen showed no positive reaction, as well as samples with concentration of antigen of $10^{5} \sim 10^{6} \mathrm{cfu} / \mathrm{ml}$ showed non-specific reactions on the test line. As a result, this experiment found that rapid kit could not detect the Lawsonia intracellularis at the pig farm.

(4) Hemorrhagic PPE can be diagnosed early when the hemoglobin is detected in the feces. Therefore, FOB test kit manufactured by Young-dong Co., ltd was used to detect hemoglobin in the feces. FOB test with 200 pretreated fecal samples from the PPE diagnosed swine by PCR test agreed $90 \%$ with the test results of PCR test. Field test showed the same results. A pair of hemoglobin was observed in the FOB kit with an electron microscope. However, $2^{\text {nd }}$ experiment with samples from a slaughter house showed $83 \%$ concordance rate with the result of PCR test in PCR positive samples. It also showed $56 \%$ non-specific response with the result of PCR test in PCR negative samples. $3^{\text {rd }}$ experiment with samples from three pig farms showed $42 \%$ concordance rate in PCR positive samples and $6 \%$ nonspecific response in PCR negative samples. The non-specific response in the negative samples was low, but the concordance rate of positive samples was also low. For this reason, it was difficult to derive a clear correlation between the positive or negative to PPE and detection of fecal occult blood.

(5) From the rapid kit test results, Lawsonia intracellularis detection was possible by the bio sensor using gold electrode and the rapid kit using polyclonal antibody and monoclonal antibody. However, those were unpractical in that impedance bio sensor cost a lot and rapid test kit showed high non-specific response with unpretreated feces. From the FOB test results, the hemorrhagic PPE was diagnosed in a single pig farm, but the concordance rate and non-specific response were high according to the pig farms. For this reason, it could not be applied in the field. Results of this study could be used for 
preliminary screening of various swine diseases. Moreover, further research is needed for lowering the non-specific response with the rapid kit.

\section{Conflict of Interest}

No potential conflict of interest relevant to this article was reported.

\section{Acknowledgement}

This research was supported by "Research Program for Agricultural Science \& Technology Development (Project No. PJ006412)", National Academy of Agricultural Science, Rural Development Administration, Republic of Korea.

\section{References}

Chung, H. S. 2008. Development of rapid diagnostic method for porcine proliferative enteropathy and characterization of Lawsonia intracellularis isolated from pigs in Korea. Doctoral Dissertation. Gyeongsang National University (In Korean).

Guedes, R. M. C., Gebhart and C. J., 2003. Preparation and characterization of polyclonal and monoclonal antibodies against Lawsonia intracellularis. Journal of Veterinary Diagnostic Investigation 15:438-446.

Young In Frontier co., ltd. 2010. ELISA test protocol. Available at: http://www.abfrontier.com/ support/ elisa.do

Kim, G., G. Yang and S. R. Lee. 2011. Rapid detection kit for Salmonella typhimurium. Journal of Biosystems Engineering 36(2):140-146 (In Korean, with English abstract).

Kim, H. J., J. T. Hong, B. K. Yu, G. Y. Kim, J. J. Lee and S. Kim. 2012. Development of rapid diagnosis technology for porcine proliferative enteropathy (1) - Preparation of the samples and antibody for rapid detecting the lawsonia in pig feces. Journal of Biosystems Engineering 37(6):420-428 (In Korean, with English abstract). 